\title{
Réorganisation du conduit vocal et réorganisation corticale de la parole : de la perturbation aux lèvres à la glossectomie. Études acoustiques et IRMf.
}

\author{
Christophe Savariaux ${ }^{1}$, Coriandre Vilain ${ }^{1}$, Monica Baciu ${ }^{2}$, Christian Abry ${ }^{1}$, Pascal Perrier ${ }^{1}$, Jacques Lebeau ${ }^{3}$, \\ Christoph Segebarth ${ }^{4}$, \\ 1- Institut de la Communication Parlée, \\ Université Stendhal, BP 25, 38040 Grenoble Cedex 9 \\ \{cvilain, perrier, savariaux,abry\}@icp.inpg.fr \\ 2 - Laboratoire de Psychologie et de NeuroCognition \\ Université Pierre Mendès-France, BP 47, 38040 Grenoble Cedex 9 \\ monica.baciu@upmf-grenoble.fr \\ 3-Service de Chirurgie Maxillo-faciale, \\ CHU Grenoble, BP 217, 38043 Grenoble Cedex 9 \\ JLebeau@chu-grenoble.fr \\ 4- Unité Mixte INSERM/UJF 594 - Neuroimagerie Fonctionnelle et Métabolique \\ CHU Grenoble, BP 217, 38043 Grenoble Cedex 9 \\ Christoph.Segebarth@ujf-grenoble.fr
}

\section{Introduction}

Le 27 novembre 2005, la première greffe humaine du visage, du nez au menton, lèvres comprises, a été à juste titre spectaculaire (et d'autres le sont devenues depuis ou le deviendront bientôt). À sa première présentation à la presse, le 6 février 2006, Isabelle Dinoire annonçait en passant, comme un bon signe de son moral, qu'elle avait recommencé à fumer (certes contre l'avis de ses médecins !) : « ...mais je fume pas clope sur clope !». Ses lèvres, qui se ferment sur une cigarette, ne peuvent pas encore vraiment se fermer pour réaliser l'occlusive bilabiale [p] (elle la produit comme un ventriloque). « Les mouvements de la lèvre inférieure ne sont pas encore présents [...]. Par conséquent la fermeture complète des lèvres ne peut encore être réalisée et bien que très en progrès, la phonation [comprendre l'articulation: son larynx n'ayant pas été endommagé] n'a pas encore les phonèmes occlusifs labiaux. A 4 mois, il est clairement trop tôt pour évaluer la récupération du mouvement.» (traduit de Devauchelle et al., 2006, p. 207). Les phonéticiens sauront être patients pour guetter les signes comportementaux de l'intégration de cette première allogreffe des lèvres, un couple d'articulateurs dont les fonctions sont très différenciées dans les diverses habiletés de la parole. Y aura-t-il, suite à cette réhabilitation, un suivi de la plasticité corticale ? On se souvient que ce fut fait pour la greffe des mains (Giraux et al., 2001). Le cas qui va nous occuper n'est pas une allogreffe de la langue, mais une greffe d'un muscle de la cuisse du sujet (le gracilis) pour opérer une reconstruction de sa langue, ce qui est effectivement un geste chirurgical bien maîtrisé. Ce qui sera pour nous spectaculaire dans ce cas, sera la récupération, mise en évidence aux niveaux comportemental et cortical, du contrôle des postures linguales des voyelles du sujet après neuf mois. Un beau succès de l'équipe de soins, 
et un succès confirmé pour la parole par l'expertise articulatoire, acoustique et neurale de l'équipe CNRS de l'Institut de la Communication Parlée en collaboration avec l'INSERM.

Que se passe-t-il au niveau comportemental, dans le conduit vocal, et conjointement au niveau cortical, lorsque les configurations du conduit vocal, qui servent à produire les sons de la parole, sont perturbées simplement mécaniquement, de manière temporaire, ou plus structurellement par un acte chirurgical ? La langue et les lèvres servent typiquement, dans le cas des voyelles, à contrôler les formes des cavités qui donnent les résonances caractéristiques, les formants, pour distinguer et reconnaître les unités cognitives du système linguistique, les phonèmes. Ainsi en français la voyelle $[\mathrm{u}]$ de toux doit comporter une aire aux lèvres suffisamment petite pour ne pas être confondue avec sa voisine [o] dans taux. C'est ce [o] que l'on produira si l'on introduit entre les lèvres du sujet un tube agrandissant la sortie du conduit vocal à plus de $1 \mathrm{~cm}^{2}$ de section. Il a été démontré dans ce cas précis (Savariaux et al., 1995, 1998) que des sujets entraînés pouvaient réussir à récupérer des formants acceptables pour le $[\mathrm{u}]$, en réorganisant la position de leur langue, notamment en la reculant, comme le prédisait notre modélisation articulatori-acoustique. Pour la langue, des perturbations aussi directes sont plus difficiles à réaliser, et c'est indirectement, en perturbant la mandibule (Gay et al., 1981, 1993) ou le feedback auditif (Houde \& Jordan, 1998, Jones \& Munhall, 2002), que les équivalences motrices et acoustiques possibles ont été testées dans la littérature. C'est cette question de l'équifinalité qui est continuée dans les expériences que nous allons rapporter ci-après. Cette question place l'étude de la production de la parole, anoblie depuis 1861 par Paul Broca, au cœur des problématiques de la cognition motrice, loin du statut de stepchild (le parent pauvre ou la «Cendrillon-souillon », notre traduction) de la psycholinguistique, un statut minoré que Pim Levelt déplorait pour la production du langage par rapport à l'impact des études en majorité écrasante sur la perception.

Un cas extrême, qui se rencontre plus rarement pour les lèvres, est l'exérèse carcinologique plus ou moins complète de la langue, dite glossectomie, avec ou sans reconstruction. Dans ce dernier cas le muscle greffé n'a bien entendu pas la complexité de la musculature linguale qui rend possible la dextérité requise pour acquérir et stabiliser le système de la langue maternelle. Néanmoins nous allons présenter un cas de glossectomisé qui, malgré un espace articulatoire devenu plus réduit à l'avant du conduit vocal, a réussi au bout de 9 mois de réhabilitation l'exploit de répéter de manière acoustiquement distincte les 10 voyelles orales du français qu'il produisait avant l'opération. Le suivi sur plusieurs séances montre comment il est parvenu à remaîtriser le recul de la masse de la langue pour [u] et aussi le contrôle acoustiquement délicat de la constriction linguale pour obtenir les formants les 
plus élevés qu'il puisse produire pour un [i]. En outre les examens IRMf ont montré qu'il avait retrouvé en partie la dominance hémisphérique gauche qu'il présentait avant l'opération, une dominance que même les sujets intacts perdent, lorsqu'ils ont à compenser une perturbation temporaire du conduit vocal, notamment aux lèvres, cruciales pour la production acoustique d'une voyelle comme [u] (Baciu et al., 2000).

\section{Sujets compensant une simple perturbation temporaire aux lèvres}

Cette étude de Baciu et al. (2000) en imagerie par résonance magnétique fonctionnelle (IRMf) a été menée sur 7 sujets (ne souffrant d'aucune pathologie de l'articulation ou de la perception de la parole), pour comprendre les implications de ces perturbations du système périphérique sur le système nerveux central. Ont été mesurées les activités cérébrales des sujets pendant une tâche d'articulation en posture normale et soutenue de la voyelle $[\mathrm{u}]$, sans phonation, comparée à une articulation avec compensation linguale pour obtenir la même catégorie vocalique $[\mathrm{u}]$, après perturbation par un tube d'environ $2 \mathrm{~cm}$ de diamètre, inséré entre les lèvres.

L'étude a montré que lors de la production du [u] non compensé, l'activité cérébrale concernait principalement la région périsylvienne gauche classique du langage, allant du cortex prémoteur (BA 6) au gyrus supramarginal (BA 40), ce dernier révélant les attentes sensorielles pariétales correspondant à l'action programmée du frontal. À un moindre niveau, certaines zones dominantes dans l'hémisphère droit étaient elles aussi recrutées. Notamment le précunéus droit (BA 7), impliqué dans des tâches diverses, de mémoire verbale ou des postures du corps, en l'occurrence les postures vocaliques des articulateurs du corps de la parole, le conduit vocal. Et aussi le gyrus supramarginal droit (BA 40). Ces activations cérébrales sont représentées classiquement par un rendu en niveaux colorés sur la surface du cortex (Figure 1). L'absence d'activation de l'aire de Broca (BA 44, 45) est à remarquer. Ceci rejoint d'autres études remettant en cause le schéma classique de cette aire comme zone correspondant à la production de la parole (Wise et al., 1999). Cette absence d'activation de l'aire de Broca a été confirmée pratiquement en même temps que nous, par l'équipe d'Ackerman (Riecker et al., 2000). 


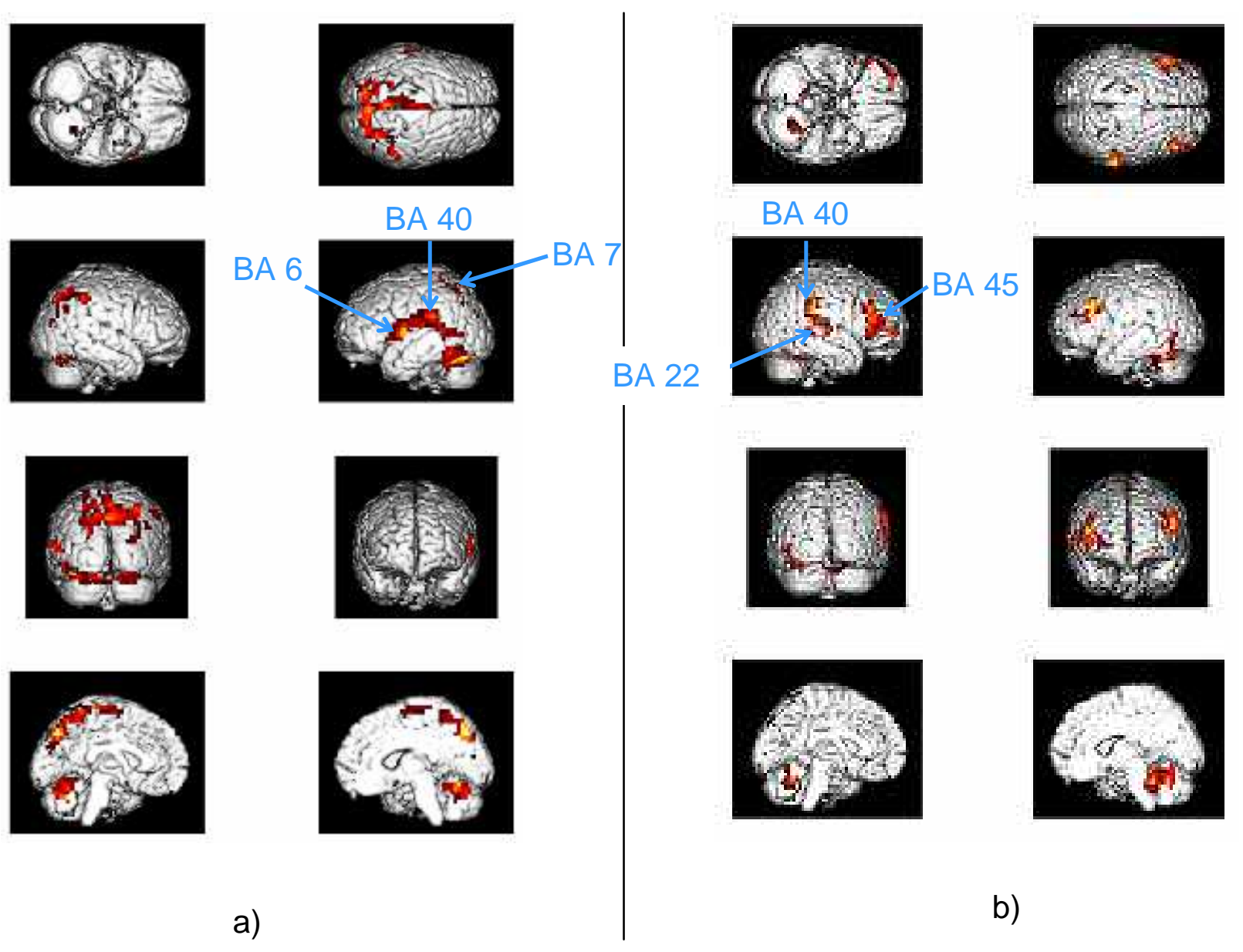

Figure 1 : Activations de la voyelle $[u]$ contrastée par rapport à la tâche de contrôle. $P$ corrigé $=0.01$. Visualisation des groupes de plus de 5 voxels. a) Articulation normale. b) Articulation compensée. Les zones cérébrales commentées dans le texte sont fléchées avec leur numéro d'aire de Brodmann (BA).

En revanche, lors de la prononciation d'un $[\mathrm{u}]$ compensé, l'activité périsylvienne gauche disparaît. Plusieurs zones de l'hémisphère droit sont alors activées, incluant en partie l'homologue droit de l'aire de Broca (BA 45), l'homologue droit de l'aire de Wernicke (BA 22), ainsi que le gyrus supramarginal droit (BA 40). Ce phénomène de changement de dominance hémisphérique dans la réalisation d'une équivalence motrice acoustique nous a donné ainsi à voir, pour la première fois, la stratégie neurale de compensation pour les sons du langage. Ce résultat de Baciu et al. (2000) sera répliqué en 2003, et intégré dans un modèle biocybernétique neural du contrôle de la parole, par Guenther et al. (2006).

L'étude de cas, que nous présentons ci-dessous, va tirer avantage des enseignements gagnés sur le comportement vocal de sujets intacts temporairement perturbé. Simplement, au lieu de considérer une perturbation mécanique, comme c'était le cas avec un tube tenu entre les lèvres par les sujets, nous serons maintenant en face d'une perturbation chirurgicale due à une pathologie, ayant nécessité une glossectomie. Ce travail se situe dans le cadre d'un projet 
général de collaboration entre le service de Chirurgie Maxillo-Faciale du CHU de Grenoble et l'ICP. Dans ce projet, nous cherchons à développer une méthodologie de caractérisation articulatoire, acoustique et cognitive de la période de réapprentissage de l'articulation de la parole, après exérèse des tumeurs cancéreuses suivie de reconstruction. L'objectif clinique à long terme est de faire évoluer les techniques de reconstruction à visée fonctionnelle facilitant en particulier le réapprentissage de la parole. Dans cette contribution, après la présentation des méthodes expérimentales utilisées, nous analyserons les résultats des examens acoustiques et ceux des examens effectués sur un patient qui s'est prêté, avec son consentement formel, à cette étude. Nous tenterons de relier performances acoustiques et activités cérébrales IRMf, à chacune des étapes préopératoire et postopératoires. Cette analyse nous permettra de proposer un schéma de la réorganisation cognitive motrice développée par le patient lors du réapprentissage de la production des sons de sa langue.

\section{Patient compensant une glossectomie après reconstruction de la langue}

Les résultats présentés dans cet article portent sur l'analyse du patient K.H., âgé de 53 ans au moment de l'opération, de sexe masculin, droitier. Le patient a été opéré suite à une tumeur cancéreuse de la langue. Il a subi une glossectomie subtotale avec une reconstruction immédiate utilisant un lambeau libre réinnervé d'un muscle pris sur l'intérieur de la cuisse, le gracilis.

\subsection{Etude acoustique}

\subsubsection{Corpus et procédure}

Le corpus est constitué de séquences sans signification (purement phonétiques) et il a été élaboré pour évaluer les capacités du patient à produire différents types d'articulation de la parole, dans des conditions variées impliquant différents degrés de contrainte temporelle (vitesse d'exécution). Dans cette contribution, nous ne traiterons que le cas de la production des voyelles isolées soutenues. Les 10 voyelles orales du français ont ainsi été enregistrées. L'ensemble du corpus a été répété 10 fois lors de chaque session d'enregistrement.

Les productions du patient ont été enregistrées sur un enregistreur numérique à bande (DAT) au CHU de Grenoble. Pour permettre une étude longitudinale et ainsi étudier l'évolution des capacités de réapprentissage articulatoires, nous avons testé le patient à 4 reprises : un enregistrement 2 jours avant l'opération pour servir de référence (condition préopératoire), un enregistrement 1 mois après l'opération (condition postopératoire 1), un enregistrement 6 mois après (postopératoire 2), et 9 mois après (postopératoire 3 ). 


\subsubsection{Analyse des données acoustiques}

Le signal acoustique a été numérisé à $20 \mathrm{kHz}$. Pour les voyelles, l'analyse est basée sur les fréquences des 3 premiers maxima du spectre de fréquences (on parle des trois premiers formants : F1, F2, F3) déterminés en analyse par prédiction linéaire. Conformément à une démarche classique (Delattre, 1948), la variation de F1 a été interprétée comme correspondant au degré d'ouverture du conduit vocal, alors que la variation de F2 a permis d'estimer la position relative avant/arrière de la langue. F3 a été exploité pour évaluer la capacité du sujet à produire des articulations extrêmes dans la région palatale. Une articulation très antérieure, avec une constriction en gouttière de la langue suffisamment courte, de type [i], correspond à un F3 élevé. La conséquence en est qu'une concentration en énergie sera produite dans la zone des hautes fréquences, la plus aiguë qui soit pour une voyelle (Schwartz et al., 1992).

\subsection{Etude IRMf}

\subsubsection{Corpus et procédure}

Nous avons restreint les enregistrements IRMf à 3 sessions. Les sessions ont eu lieu aux mêmes dates que les enregistrements acoustiques en condition préopératoire, postopératoire 1 ( 1 mois après l'opération) et postopératoire 3 ( 9 mois après l'opération).

Pour chaque enregistrement, les tâches réalisées par le patient étaient les mêmes. Elles consistaient à articuler de façon soutenue mais sans phonation les 3 voyelles cardinales [i], [a] et $[\mathrm{u}]$. Une séance d'enregistrement consistait en 3 sessions d'acquisition fonctionnelle correspondant aux 3 voyelles et une session d'acquisition anatomique. Les sessions d'acquisition fonctionnelles suivaient un paradigme de type bloc, durant lequel les tâches d'articulation alternaient avec des tâches de contrôle pendant lesquelles le sujet restait au repos.

Les enregistrements ont été effectués dans l'imageur clinique Philips NT, 1.5 Tesla du CHU de Grenoble. Cet imageur est équipé avec des techniques d'imagerie rapide de type EPI. Le volume mesuré était centré sur la limite supérieure du thalamus et orienté parallèlement au plan bi-commissural CA-CP. Le choix du volume mesuré pendant les acquisitions fonctionnelles a été effectué sur la base d'une séance de repérage anatomique à faible résolution spatiale dans le plan sagittal, en début d'examen. Lors des mesures, l'intégralité du cerveau était mesurée en 26 coupes de $5 \mathrm{~mm}$ chacune. La durée d'enregistrement d'un volume complet était de 3096 secondes, et chaque volume a été mesuré 14 fois par tâche. Chaque 
tâche durait 43 secondes, et l'alternance des tâches de contrôle et des tâches d'articulation a été répétée 4 fois dans chaque session d'acquisition.

Les principaux paramètres d'acquisition étaient l'angle de basculement $\left(90^{\circ}\right)$, le champ de vision $\left(256^{*} 256 \mathrm{~mm}^{2}\right)$, la matrice d'acquisition $(64 * 64)$, et la matrice de reconstruction $(128 * 128)$.

En fin d'examen, une acquisition anatomique générant des images à haute résolution spatiale a été réalisée, permettant de faire correspondre les zones d'activités cérébrales avec l'anatomie cérébrale propre au sujet.

\subsubsection{Déroulement des séances d'acquisition}

Le sujet était allongé dans l'aimant dans la position la plus confortable qui lui permette de rester de 30 à 40 minutes sans bouger. Par le biais d'un système de miroirs, il recevait les consignes visuelles lui indiquant la tâche à réaliser. Les consignes étaient présentées par le logiciel PsyScope V.1.1 fonctionnant sur un ordinateur Power Macintosh 9600. Elles étaient projetées sur un écran au moyen d'un vidéo-projecteur (Eiki LC600). Le système de miroir situé dans l'aimant permettait au sujet de voir cet écran. Enfin, pour atténuer le bruit généré par l'imageur lors des acquisitions, le sujet portait des bouchons d'oreille et un casque anti-bruit.

\subsubsection{Analyse des données IRMf}

Le traitement des données a été effectué grâce au logiciel SPM99 (Statistical Parametric Mapping, Friston et al., 1995). Ce traitement s'est fait en deux phases : une phase de prétraitement suivi d'une phase d'analyse statistique. Lors du prétraitement, les volumes fonctionnels acquis lors de chaque session ont d'abord été corrigés pour les décalages temporels entre chaque coupe. Ensuite, tous les volumes ont été réalignés sur le premier volume acquis pour corriger les artefacts liés aux mouvements de la tête du sujet. Après ce réalignement, les volumes ont été normalisés spatialement à l'aide d'une transformation nonlinéaire afin qu'ils puissent coïncider avec le gabarit de référence du Montreal Neurological Institute (MNI). Enfin, les scans ont été lissés spatialement avec un filtre gaussien pour limiter les corrélations entre les activités des voxels voisins.

Une fois effectuées les opérations de prétraitement, le Modèle Linéaire Généralisé a été appliqué sur chaque voxel. Ce modèle permet d'exprimer les évolutions temporelles de l'activation des voxels comme une combinaison linéaire de facteurs explicatifs à laquelle se rajoute un terme d'erreur supposé fluctuer normalement. Les inférences statistiques sont effectuées sur les coefficients multiplicatifs des facteurs explicatifs. Elles permettent de tester si un voxel est significativement plus activé par une condition expérimentale que par une 
autre. Les tests effectués sont des tests de Student utilisant comme seuil de significativité des p-valeurs, corrigées pour tenir compte des comparaisons multiples.

\section{Résultats comportementaux et cérébraux}

\subsection{Résultats de l'analyse acoustique de la production des voyelles}

Les valeurs moyennes des mesures formantiques (F1, F2, F3) obtenues lors de la production des voyelles sont rassemblées dans le Tableau 1. Dans la suite de cet article nous avons choisi de représenter dans le plan F1/F2, l'ensemble des productions vocaliques (plutôt que la moyenne associée à ces productions) : cette représentation nous donne la dispersion des productions pour chaque catégorie de voyelle. Pour les conditions postopératoires, nous focaliserons notre étude dans le plan F2/F3, sur les distinctions entre les voyelles [i] et [y] reposant sur F3 : en conséquence seules ces deux voyelles seront représentées sur les Figures ci-après.

Les voyelles produites en condition préopératoire ne présentent pas de particularité, si on les compare aux données issues de la littérature sur le français (Calliope, 1989). On peut ainsi retrouver sur la Figure 2a une distribution classique, en forme de triangle des voyelles dans le plan F1/F2. La représentation de ces mêmes voyelles dans le plan F2/F3 (Figure 2b) reste également conforme à celle de la littérature : on retrouve une distinction [i] $v s$. [y] sur la base d'un formant F3 plus élevé pour la voyelle [i] (Schwartz et al., 1992). Ces résultats nous indiquent que la tumeur du patient avant opération ne semblait pas induire de modification significative sur la qualité de ses voyelles. Nous prendrons ces valeurs fréquentielles comme références pour la suite de l'analyse acoustique. Pour situer les conditions postopératoires, nous réaliserons un zoom sur l'axe des abscisses et des ordonnées à partir du rectangle en trait tireté représenté sur ces Figures $2 \mathrm{a}$ et $2 \mathrm{~b}$ : ce sera le sous-espace de cet espace avant l'intervention, le sous-espace que le sujet aura pu récupérer en contrôlant son nouveau muscle réinnervé. 


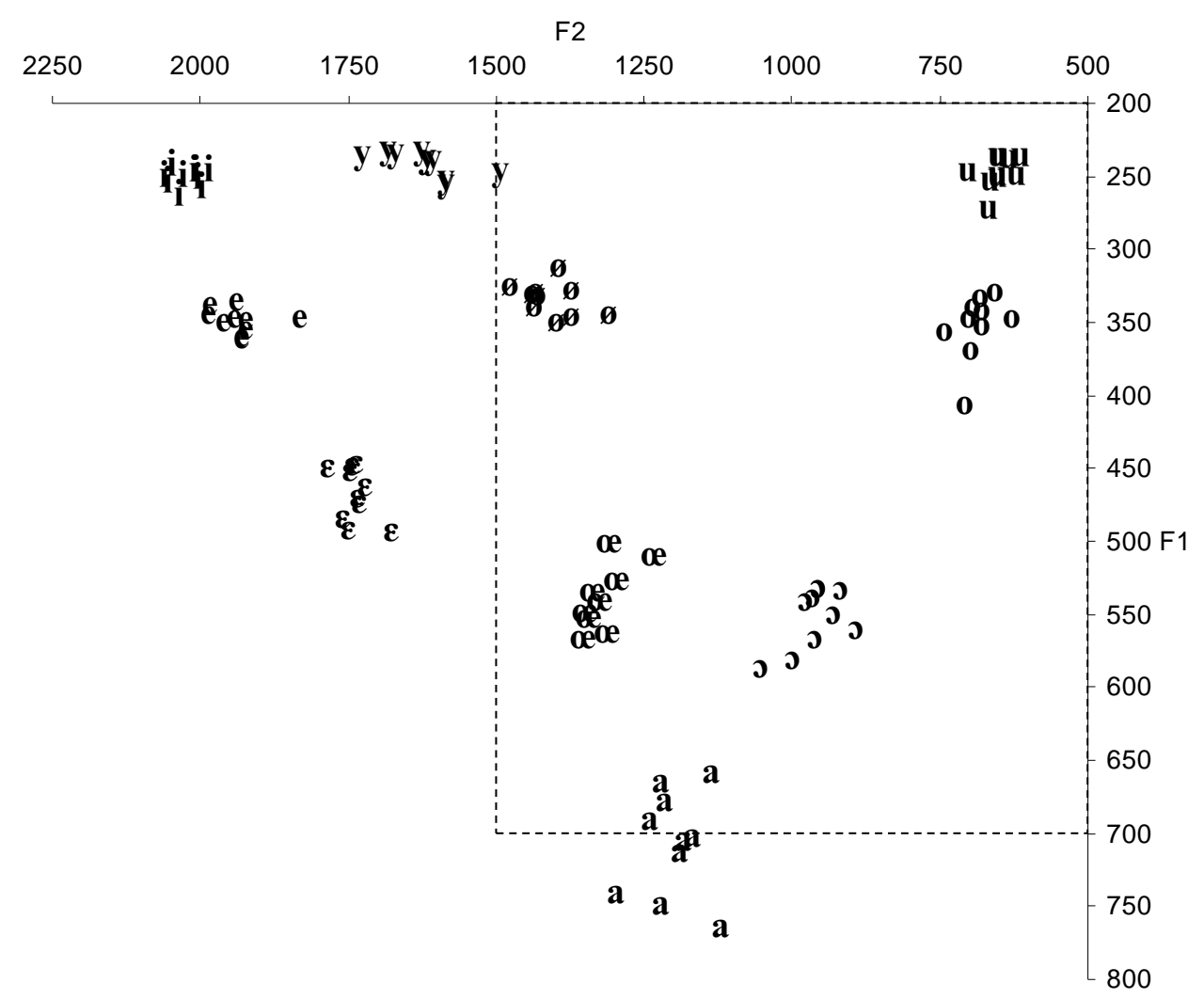

Figure 2a : Valeurs en $\mathrm{Hz}$ des 10 répétitions des 10 voyelles dans le plan F1/F2, condition préopératoire (cf. texte pour plus d'explications).

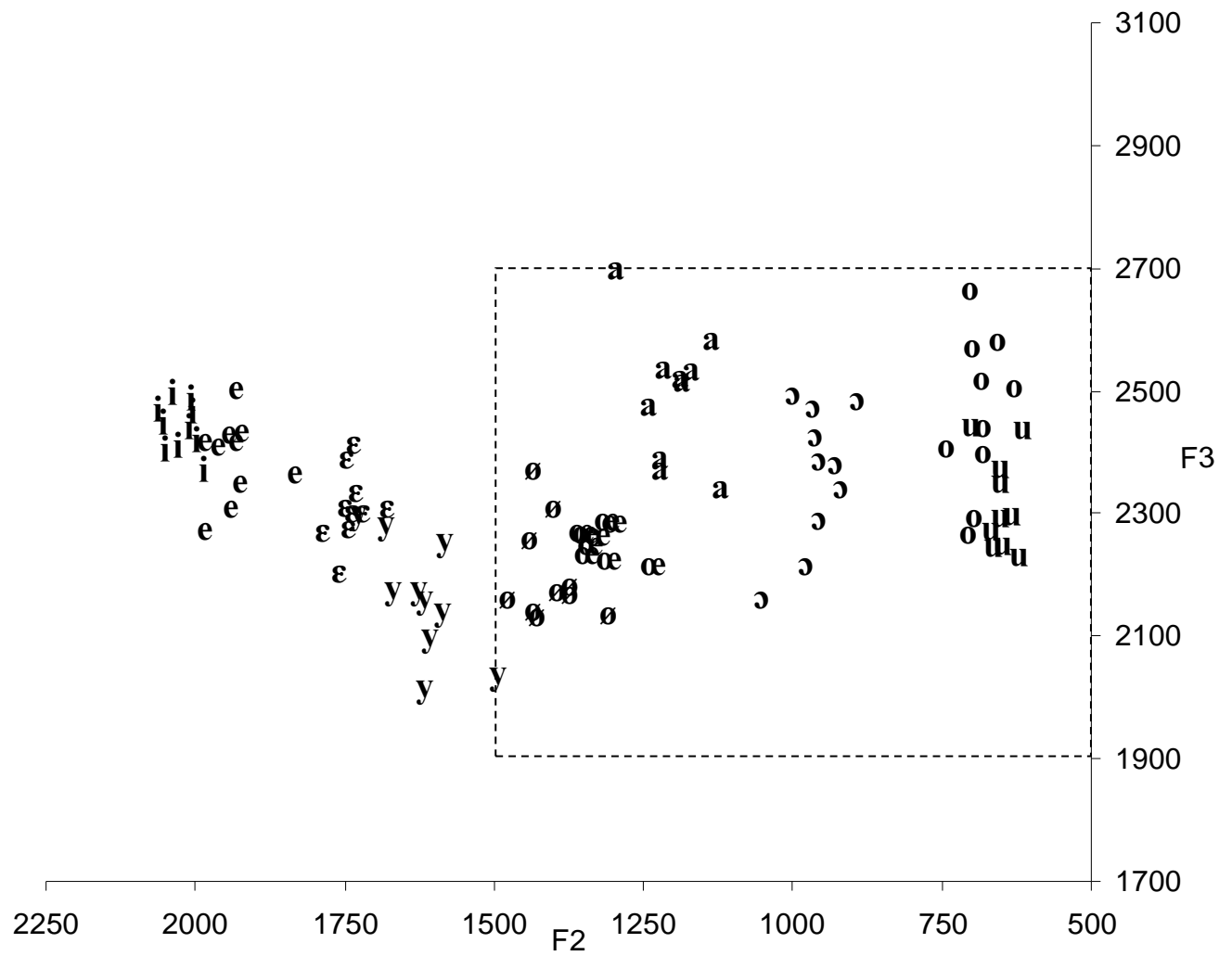

Figure 2b : Valeurs en $\mathrm{Hz}$ des 10 répétitions des 10 voyelles dans le plan F2/F3, condition préopératoire. 


\begin{tabular}{|c|c|c|c|c|c|}
\hline \multicolumn{2}{|c|}{ Voyelle } & Préopératoire & Postopératoire 1 & Postopératoire 2 & Postopératoire 3 \\
\hline \multirow{3}{*}{$\mathrm{i}$} & F1 (std) & $251(6)$ & $311(12)$ & $264(19)$ & $249(16)$ \\
\hline & F2 (std) & $2024(25)$ & $1169(28)$ & $1147(12)$ & $1179(19)$ \\
\hline & F3 (std) & $2438(38)$ & $2541(62)$ & $2495(57)$ & $2423(35)$ \\
\hline \multirow{6}{*}{$\mathrm{e}$} & F1 (std) & $349(8)$ & $400(15)$ & $396(26)$ & $403(24)$ \\
\hline & F2 (std) & $1937(41)$ & $1213(43)$ & $1210(28)$ & $1275(43)$ \\
\hline & F3 (std) & $2390(65)$ & $2468(48)$ & $2550(45)$ & $2513(42)$ \\
\hline & F1 (std) & $467(17)$ & $569(21)$ & $523(27)$ & $554(27)$ \\
\hline & F2 (std) & $1741(26)$ & $1163(36)$ & $1222(19)$ & $1294(48)$ \\
\hline & F3 (std) & $2310(56)$ & $2421(23)$ & $2479(27)$ & $2491(47)$ \\
\hline \multirow{3}{*}{$\mathrm{a}$} & F1 (std) & $707(34)$ & $651(16)$ & $640(15)$ & $672(18)$ \\
\hline & $\mathrm{F} 2(\mathrm{std})$ & $1200(49)$ & $1006(35)$ & $1003(26)$ & $1001(23)$ \\
\hline & F3 (std) & $2495(102)$ & $2338(27)$ & $2478(26)$ & $2435(23)$ \\
\hline \multirow{3}{*}{$\mathrm{y}$} & F1 (std) & $240(8)$ & $238(12)$ & $241(20)$ & $236(15)$ \\
\hline & F2 (std) & $1621(61)$ & $1158(53)$ & $1137(19)$ & $1152(17)$ \\
\hline & F3 (std) & $2253(223)$ & $2372(68)$ & $2439(93)$ & $2343(37)$ \\
\hline \multirow{3}{*}{$\varnothing$} & F1 (std) & $334(11)$ & $307(9)$ & $332(17)$ & $343(25)$ \\
\hline & $\mathrm{F} 2$ (std) & $1407(45)$ & $1154(31)$ & $1152(20)$ & $1104(22)$ \\
\hline & F3 (std) & $2202(77)$ & $2329(46)$ & $2366(70)$ & $2304(34)$ \\
\hline \multirow{3}{*}{$œ$} & F1 (std) & $539(21)$ & $528(16)$ & $514(30)$ & $525(26)$ \\
\hline & F2 (std) & $1319(35)$ & $1086(31)$ & $1061(21)$ & $1045(36)$ \\
\hline & F3 (std) & $2254(24)$ & $2349(46)$ & $2367(22)$ & $2323(44)$ \\
\hline \multirow{3}{*}{$\mathrm{u}$} & F1 (std) & $246(11)$ & $255(7)$ & $303(30)$ & $316(22)$ \\
\hline & F2 (std) & $653(24)$ & $884(60)$ & $729(59)$ & $680(40)$ \\
\hline & F3 (std) & $2318(74)$ & $2138(45)$ & $2211(57)$ & $2145(45)$ \\
\hline \multirow{3}{*}{$\mathrm{o}$} & F1 (std) & $353(21)$ & $330(10)$ & $373(17)$ & $397(20)$ \\
\hline & F2 (std) & 688 (29) & $653(49)$ & $632(68)$ & $595(46)$ \\
\hline & F3 (std) & $2463(121)$ & $2178(47)$ & $2252(67)$ & $2199(20)$ \\
\hline \multirow{3}{*}{0} & F1 (std) & $552(19)$ & $581(25)$ & $571(31)$ & $547(41)$ \\
\hline & F2 (std) & $963(42)$ & $892(46)$ & $742(45)$ & $748(32)$ \\
\hline & F3 (std) & 2364 (108) & $2262(22)$ & $2319(36)$ & $2285(42)$ \\
\hline
\end{tabular}

Tableau 1: Valeurs moyennes en $\mathrm{Hz}$ des 3 premiers formants sur les 10 répétitions des 10 voyelles orales françaises du patient K.H. (avec l'écart-type correspondant entre parenthèses). 
La distribution des voyelles produites en condition postopératoire 1, après un mois, atteste d'une grande difficulté pour le patient à reproduire l'espace classique des voyelles dans le plan F1/F2 (Figure 3a). On remarquera ainsi que les valeurs de F2 vont rester confinées endeçà de $1500 \mathrm{~Hz}$ environ (valeur qui ne sera approchée qu'au bout de neuf mois et pour quelques exemplaires des voyelles, cf. Figure 5a) : ces valeurs attestent d'une réduction très nette de l'amplitude du mouvement avant/arrière de la langue. Les voyelles [i] et [u] sont les plus touchées par cette réduction. Le patient semble en revanche capable de conserver des valeurs de F1 du même ordre de grandeur que celui des productions en condition préopératoire: globalement il préserve la hiérarchie du degré d'aperture (ouverture) du conduit vocal. Mais il n'arrive pas à élever la langue assez haut pour produire la voyelle [i] et la voyelle [u]. En ce qui concerne le plan F2/F3 (Figure 3b), les valeurs de F3 obtenues pour la voyelle [i] sont presque toutes supérieures à celles de la voyelle [y], permettant ainsi au patient de garder une distinction entre ces deux voyelles sur la base des fréquences de ce formant haut. Le patient, un mois après l'opération, se trouve donc dans l'incapacité de réaliser un geste lingual d'amplitude équivalente à celui produit en condition préopératoire, et ainsi de produire une distinction entre plusieurs voyelles d'avant, notamment autour de [i] et de $[œ]$.

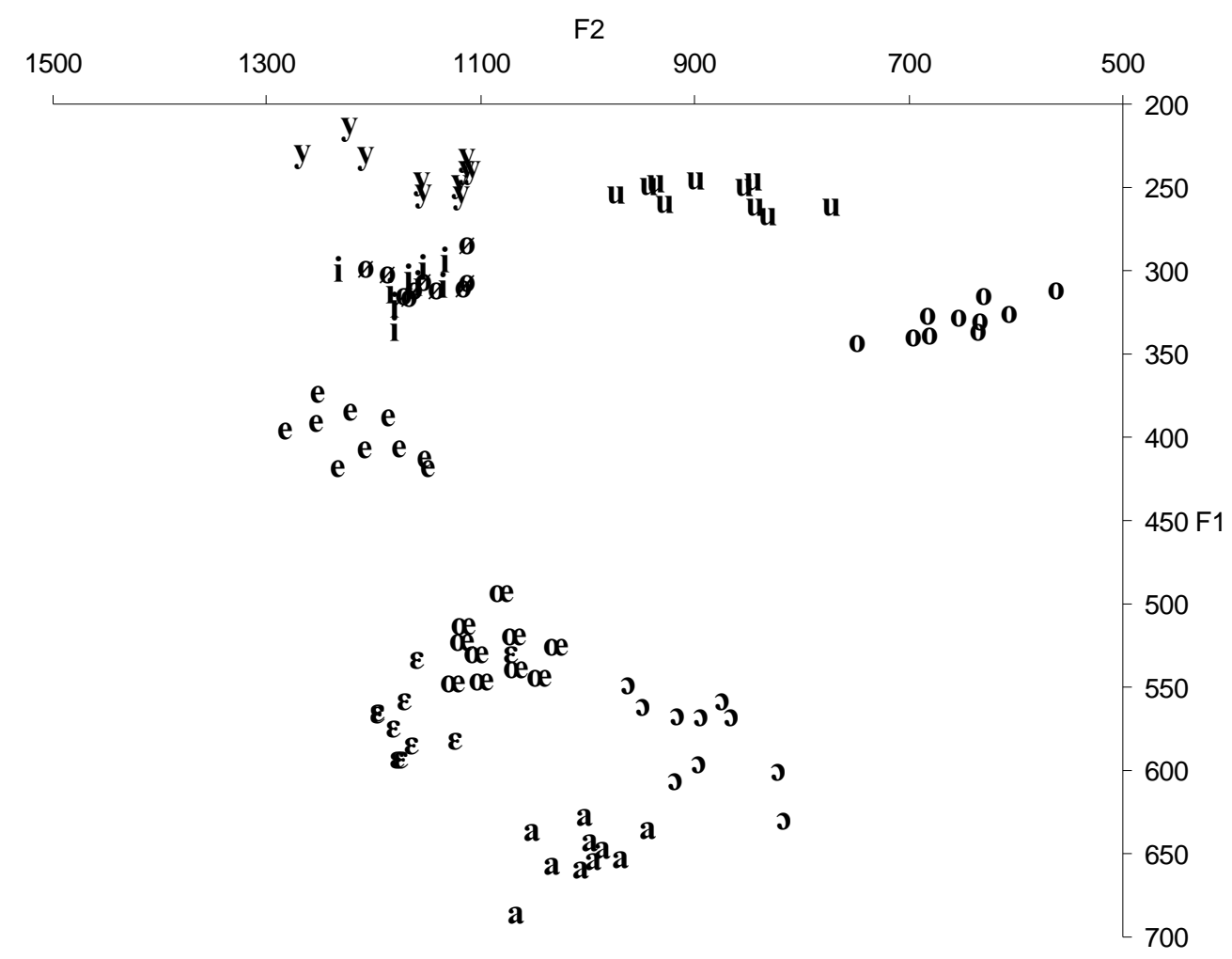

Figure 3a : Valeurs en $\mathrm{Hz}$ des 10 répétitions des 10 voyelles dans le plan F1/F2, condition postopératoire 1 . 


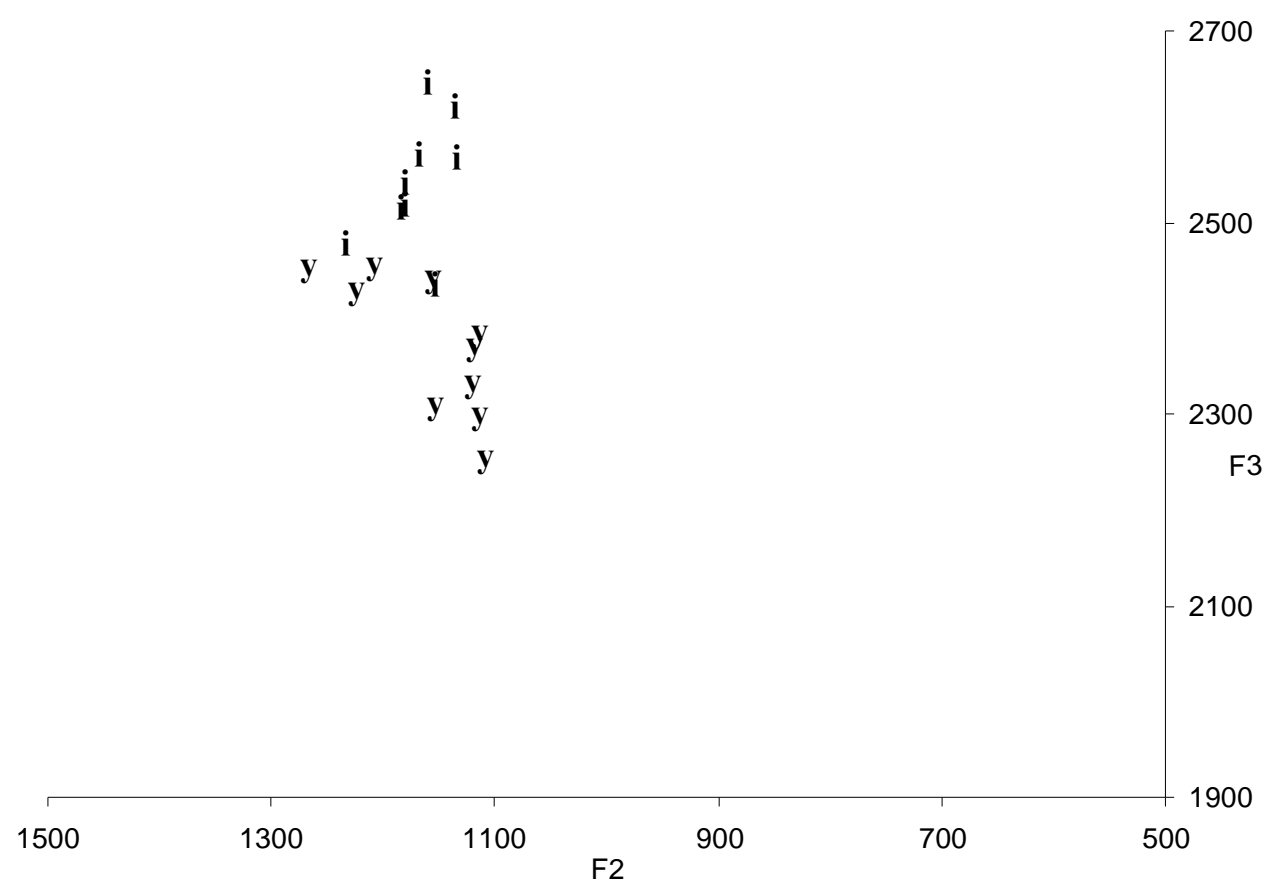

Figure 3b : Valeurs en $\mathrm{Hz}$ des 10 répétitions des voyelles [i] et [y] dans le plan F2/F3, condition postopératoire 1.

Si on compare l'espace vocalique obtenu 6 mois après l'opération, en condition postopératoire 2 (Figure 4a), à celui obtenu en condition postopératoire 1, deux tendances émergent : (i) l'amplitude de variation du formant F2 et donc du mouvement avant/arrière de la langue est aussi restreinte dans les deux cas; (ii) globalement une différenciation plus marquée pour les voyelles qui étaient proches en condition postopératoire 1 émerge en condition postopératoire 2. On a maintenant une voyelle [i] avec des valeurs de F1 standards : le patient est donc plus capable de serrer la constriction de cette voyelle. On peut également noter qu'à ce stade (condition postopératoire 2), il parvient à réaliser un geste lingual plus reculé que précédemment pour la voyelle $[\mathrm{u}]$ (qui en conséquence se rapproche naturellement de la voyelle [o], sans pour autant se confondre acoustiquement avec elle). Ceci se traduit par une valeur moyenne de F2 plus faible $(729$ vs. $884 \mathrm{~Hz})$ et donc plus proche de la valeur standard. Dans le plan F2/F3, sur la base des valeurs de F3, la distinction entre les voyelles [i] et [y] est maintenant brouillée par rapport à la condition postopératoire 1 (Figure 4b). En résumé, l'espace des voyelles produites par le patient 6 mois après l'opération reste réduit à l'avant. Mais on note cependant une différenciation globalement bien mieux réalisée entre les voyelles que celle obtenue après un mois. La voyelle [i] est ainsi produite avec des valeurs de F1 proche des valeurs standards comme c'est aussi le cas de la voyelle [u] en F1 et F2, ce qui dénote que le patient a retrouvé plus de mobilité pour produire les voyelles hautes. 


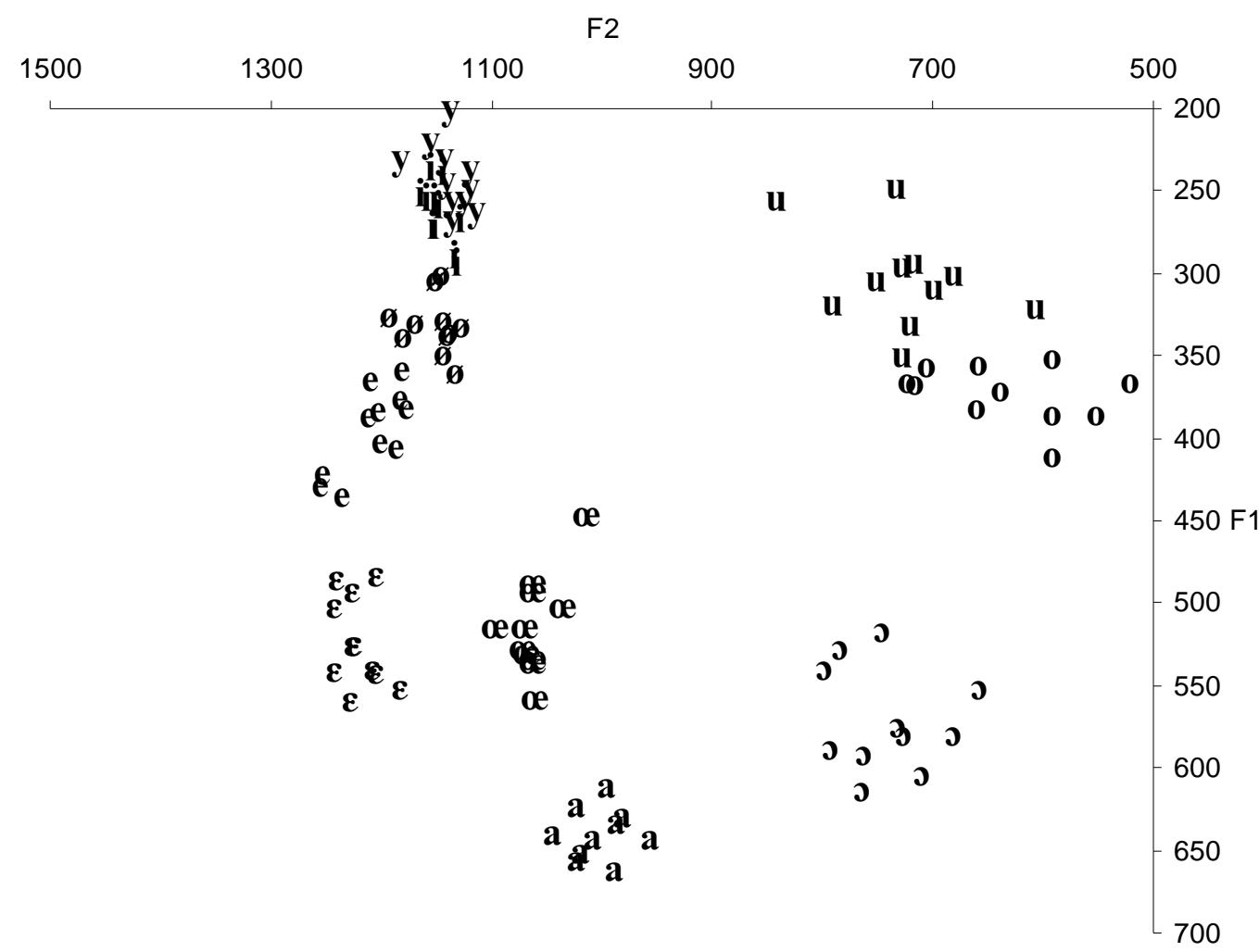

Figure 4a : Valeurs en $\mathrm{Hz}$ des 10 répétitions des 10 voyelles dans le plan F1/F2, condition postopératoire 2.

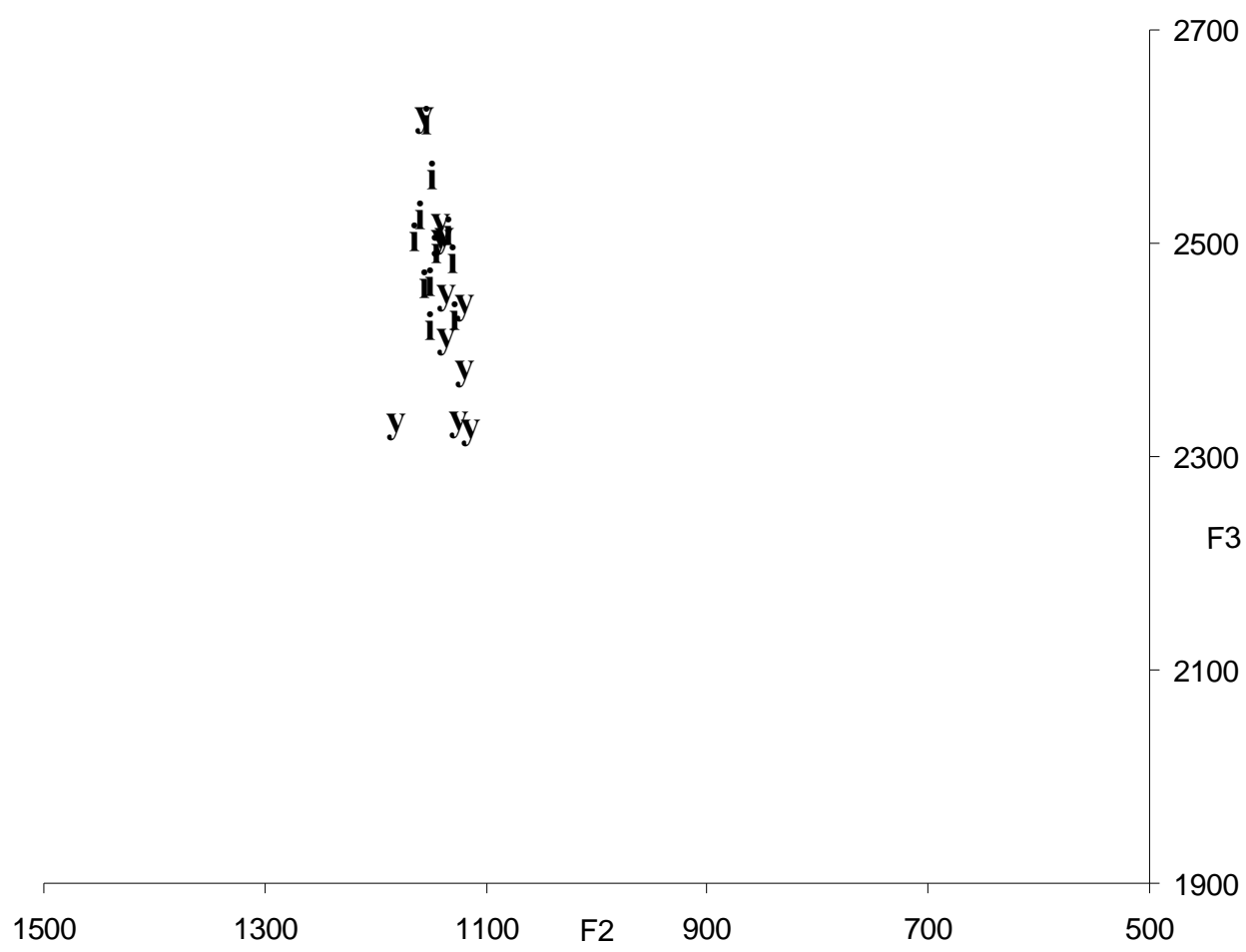

Figure 4b : Valeurs en $\mathrm{Hz}$ des 10 répétitions des voyelles [i] et [y] dans le plan F2/F3, condition postopératoire 2. 
La distribution des voyelles obtenue par le patient 9 mois après son opération, en condition postopératoire 3 (Figure 5a), montre que son espace vocalique, tout en étant encore loin de son espace préopératoire, s'est légèrement agrandi vers l'avant. Et surtout cela correspond à une meilleure distinction des voyelles d'avant. Au total il a retrouvé, dans un espace certes plus réduit, toutes les distinctions de ses voyelles du français. L'exception des voyelles [i] et [y], qui restent identiques dans le plan F1/F2, n'en est pas une, puisque dans le plan F2/F3 les deux séries de voyelles sont séparées, un seul des dix [y] se retrouvant au milieu des [i] (Figure 5b). On peut donc affirmer quantitativement que le patient aura réussi à recatégoriser acoustiquement $99 \%$ de ses voyelles orales du français. L'évaluation perceptive sur les qualités de ces productions, qui pourront être jugées en partie non encore conformes à la norme du français, ne doit pas court-circuiter l'étape nécessaire de cette évaluation articulatori-acoustique sur l'efficacité du contrôle de l'effecteur reconstruit, pour sa performance biosonique à distinguer 10 catégories de signaux de la langue maternelle du sujet.

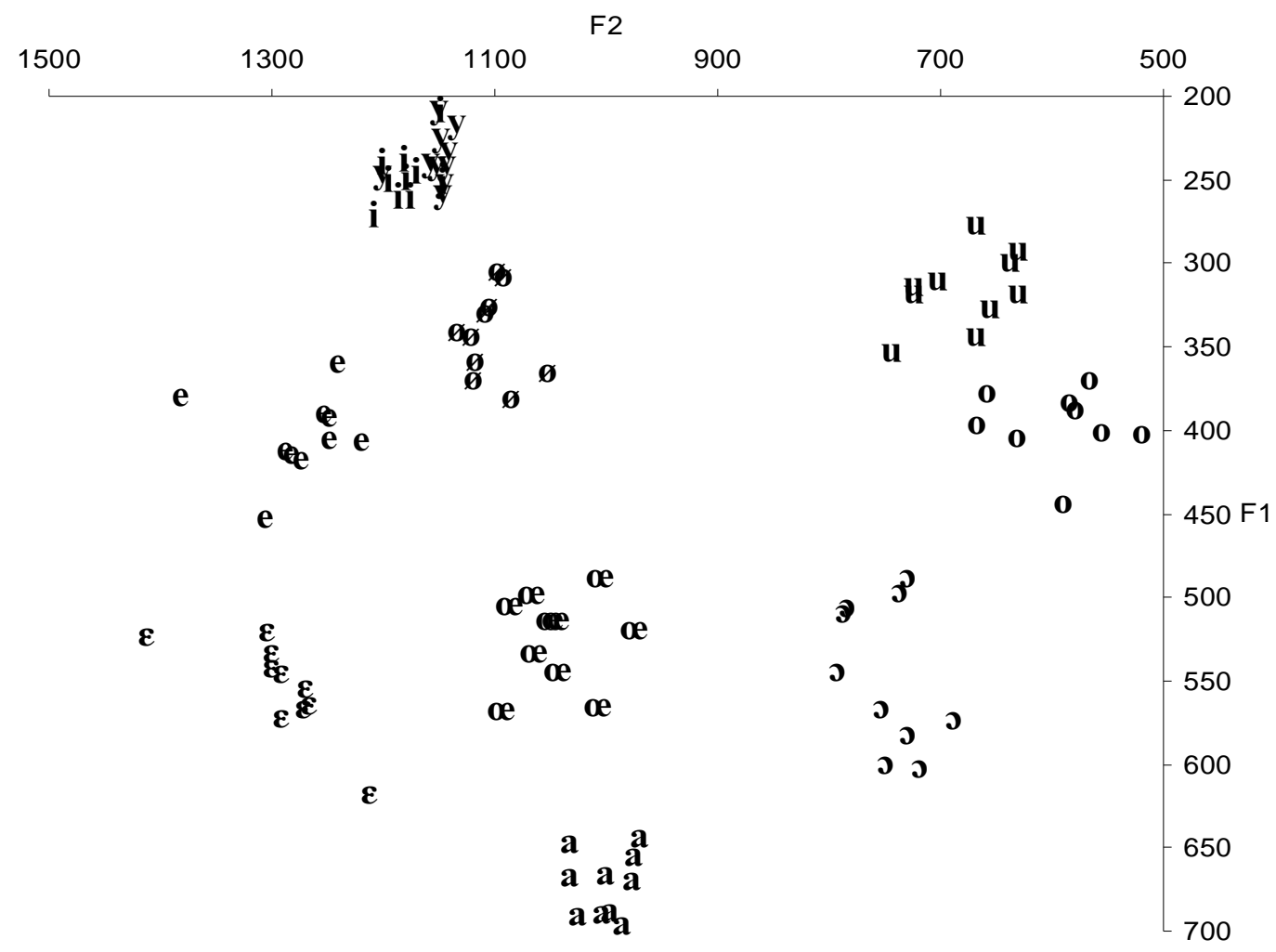

Figure 5a : Valeurs en $\mathrm{Hz}$ des 10 répétitions des 10 voyelles dans le plan F1/F2, condition postopératoire 3 . 


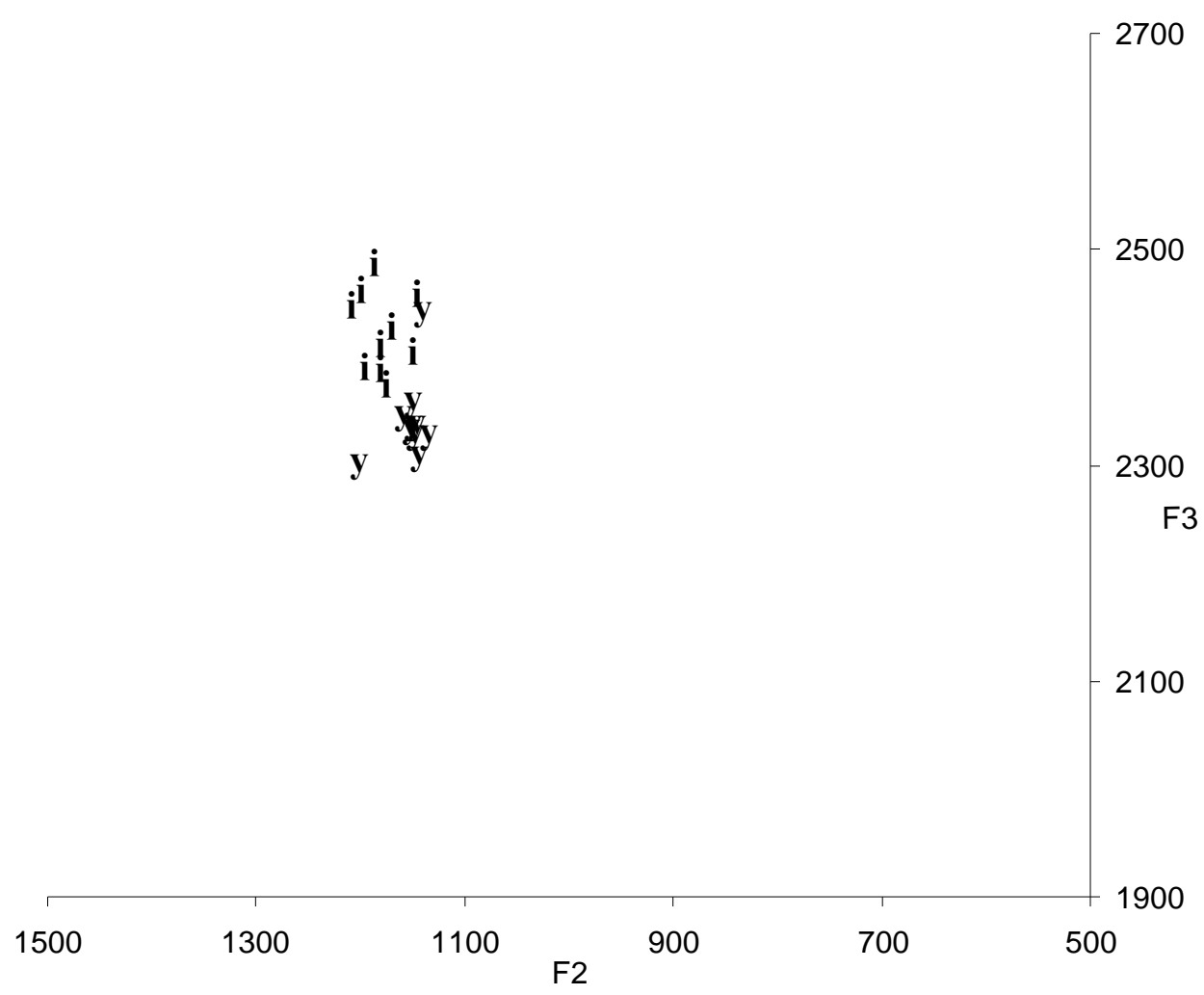

Figure 5b : Valeurs en Hz des 10 répétitions des voyelles [i] et [y] dans le plan F2/F3, condition postopératoire 3 .

\subsection{Résultats de l'analyse IRMf}

Dans un premier temps nous avons mesuré les différences d'activation entre les phases d'articulation et les phases de repos de l'ensemble des voyelles. La Figure 6 présente l'évolution des activations cérébrales correspondant à l'articulation des 3 voyelles, aux stades préopératoire (à gauche), et postopératoires 1 (au centre) et 3 (à droite). Il est possible de distinguer 4 zones principales d'activation: le pôle temporal (BA 38), bilatéralement, le cervelet, aussi bilatéralement, le cortex périsylvien gauche autour de l'insula (BA 13), et le cortex prémoteur gauche autour de l'aire BA 6.

Nous pouvons noter l'activité du pôle temporal bilatéral (BA 38) lors des trois séances de mesure. Sachant que cette zone est reliée à l'insula et au cortex auditif associatif (partie antérieure du Gyrus Temporal Supérieur), il se pourrait que le sujet l'active en fonction des attentes auditives de son action articulatoire (Gloor, 1997). 

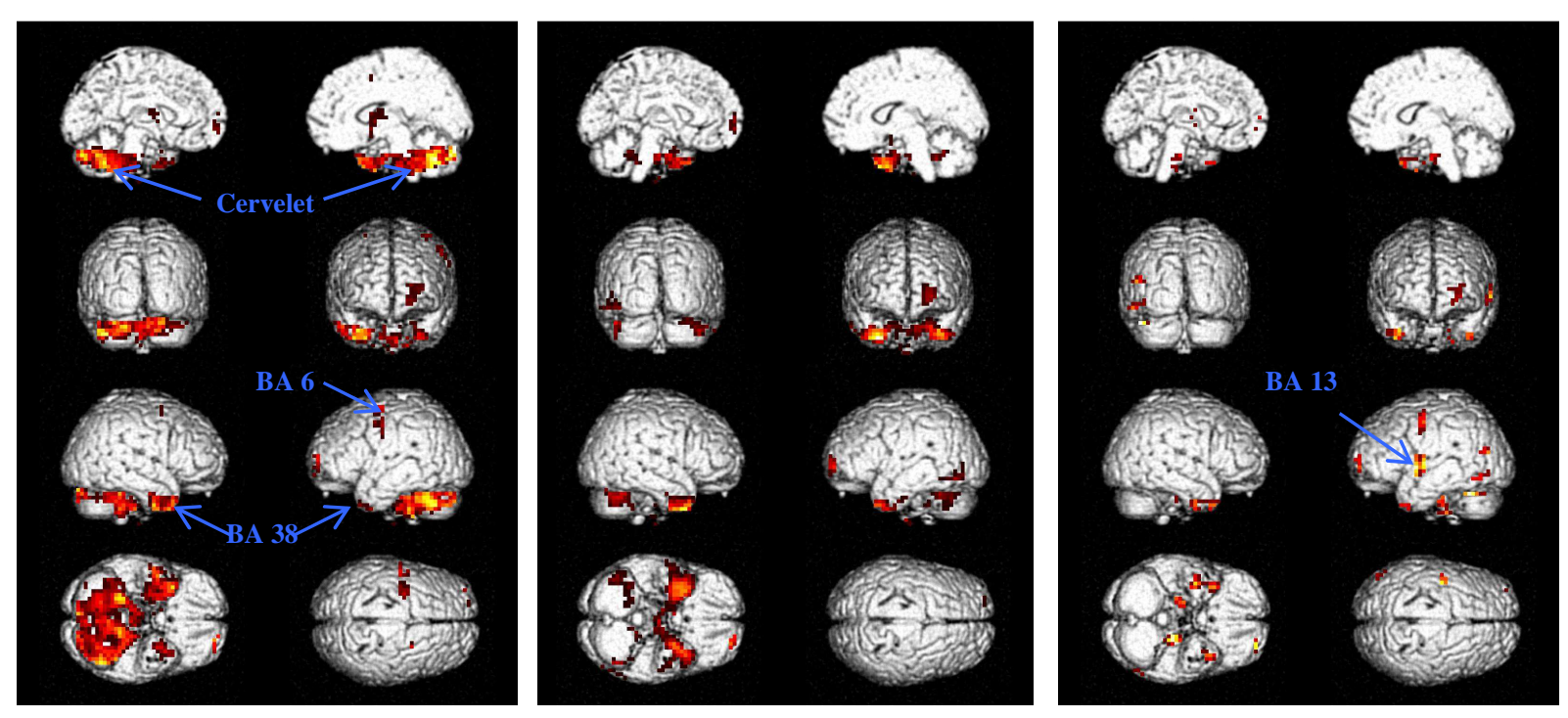

Figure 6 : Evolution des activations pour l'articulation des 3 voyelles [i], [a] et [u], relativement à la tâche de contrôle, depuis le stade préopératoire (à gauche), jusqu'aux stades postopératoires 1 (après 1 mois, au centre) et 3 (après 9 mois, à droite). $P$ corrigé = 0.01. Affichage des groupes d'au moins 10 voxels. Les zones cérébrales commentées dans le texte sont fléchées.

Le cervelet est une zone connue pour son rôle dans la coordination motrice. Il entre dans la synchronisation des mouvements lors de tâches motrices, y compris pour l'articulation de la parole (voir une revue de question dans Ivry et al., 2001). Dans nos mesures, l'activation du cervelet est bilatérale. Elle est importante lors de l'enregistrement préopératoire et devient faible puis négligeable lors des enregistrements postopératoires 1 et 3. Une piste pour expliquer ce comportement pourrait être que le cervelet est plus activé lors de tâches automatiques n'ayant que peu besoin de recruter les zones du cortex non cérébelleux. Dans la production de la parole, il n'est sans doute pas nécessaire de recruter l'aire de Broca pour l'articulation (Wise et al., 1999), ici pour celle d'une simple voyelle : c'est une tâche qui peut être considérée comme hautement automatique, ce que traduit une activité importante du cervelet lors de l'enregistrement préopératoire. En revanche, suite à la désorganisation articulatoire post-opératoire, la parole serait désautomatisée et recruterait d'autres zones du cortex.

En ce qui concerne l'activité des zones de l'hémisphère gauche appartenant aux aires traditionnelles du langage, sont recrutés principalement le cortex insulaire gauche (BA 13) et le cortex prémoteur gauche (BA 6). Les activations du cortex prémoteur gauche (BA 6) sont 
connues pour la programmation du geste articulatoire. Nous pouvons observer que cette zone est activée significativement lors des enregistrements préopératoire et postopératoire 3. Elle ne l'est pas lors de l'enregistrement postopératoire 1. Il est possible que la perturbation postopératoire modifie en profondeur les stratégies cognitives d'articulation des zones recrutées pour cette action, ce qui expliquerait la disparition de l'activation classique de l'hémisphère gauche lors de l'enregistrement postopératoire 1. Alors qu'après la phase de réapprentissage, en postopératoire 3, l'activité normale du cortex prémoteur réapparaît, comme semble l'attester les résultats obtenus sur les voyelles montrant qu'en condition postopératoire 3 le patient a trouvé une stabilité dans ses productions vocaliques : il n'est plus en phase nouvelle de réapprentissage.

Ce retour de l'activation du cortex prémoteur dans l'enregistrement postopératoire 3 s'accompagne d'une activité très marquée du cortex insulaire gauche (BA 13). L'importance de ce dernier en parole a été mise en avant par de nombreux travaux (Dronkers, 1996, Bennett \& Netsell, 1999, Ackerman \& Riecker, 2004). Dronkers, en particulier, lors de ses travaux sur l'apraxie de la parole, a montré que parmi 25 patients ayant un déficit de planification articulatoire, consécutif à un accident vasculo-cérébral de l'hémisphère gauche, la zone du cortex insulaire précentral gauche était systématiquement lésée. Elle en a déduit le rôle central de cette aire pour les tâches de planification motrice dans la parole (Dronkers, 1996). Depuis, Hillis et al. (2004) ont modéré ses conclusions en montrant que les patients suivis par Dronkers avaient eu leur accident vasculaire plusieurs mois avant l'étude et que des modifications anatomiques pouvaient avoir eu lieu durant cette période (cf. pourtant Ogar et al., 2006, dont Dronkers, qui réaffirment ses conclusions de 1996). Enfin, Ackermann \& Riecker (2004) ont eux aussi pointé le rôle central de l'insula lors de l'exécution de la parole. En ce qui nous concerne, il est surprenant de constater que si l'activation de l'insula est importante pour la planification ou l'exécution de la parole, elle n'est activée que lors de l'enregistrement postopératoire 3. Si l'on fait abstraction de l'enregistrement postopératoire 1, pour lequel les stratégies articulatoires semblent très désorganisées, il semblerait logique d'observer une activation de l'insula en enregistrement préopératoire. Or, même si l'on considère spécifiquement l'activation liée à l'articulation de toutes les voyelles lors de l'enregistrement préopératoire, relativement à l'enregistrement postopératoire 1, on n'obtient pas d'activation de l'insula (Figure 7). Nous pouvons donc mettre en avant une autre explication concernant l'activation de l'insula. Nous proposons que l'insula soit activée lors de la tâche de réautomatisation du geste articulatoire et que, lorsque ce geste est automatisé, le cervelet reprend ce contrôle. 


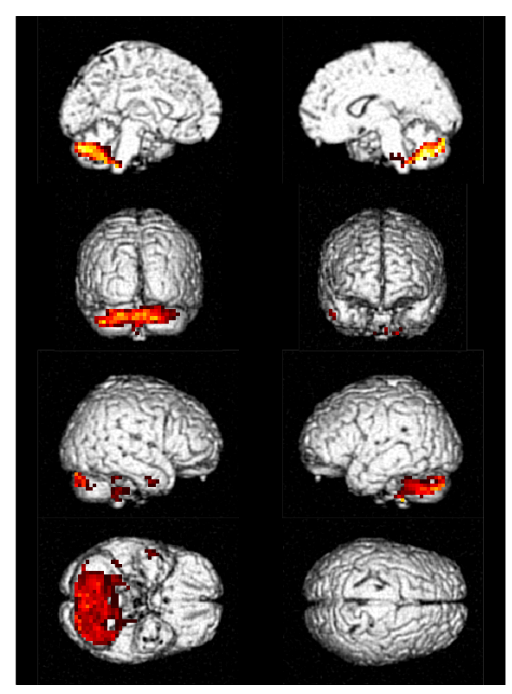

Figure 7 : Activations liées à l'articulation de toutes les voyelles en enregistrement préopératoire relativement à l'enregistrement postopératoire 1 . $P$ corrigé $=0.05$. Affichage des groupes d'au moins 5 voxels.

\section{Conclusions}

Nous avons étudié le cas d'un patient ayant subi une opération de glossectomie suivie d'une reconstruction de la langue. Le suivi de ses productions acoustiques a démontré au bout de 9 mois une récupération remarquable, à $99 \%$, des 10 distinctions phonémiques vocaliques orales du français. L'enregistrement suivi de ses activités cérébrales, lors des tâches d'articulation, nous a permis de proposer un schéma cognitif pour la production des voyelles, à la lumière des résultats précédents sur les sujets intacts soumis à une perturbation configurationnelle de leur conduit vocal (Baciu et al., 2000). Ce schéma est le suivant.

Lors de l'enregistrement préopératoire, l'articulation des voyelles est une habileté cognitive tout à fait automatisée; elle recrute massivement chez le sujet le cervelet et le cortex prémoteur gauche.

Juste un mois après l'opération, l'articulation d'une voyelle devient une tâche complexe nécessitant de modifier les stratégies habituelles. L'activité cérébrale est alors diffuse et aucune zone n'émerge vraiment. Chez les sujets intacts aussi, quand on perturbe leur conduit vocal, la dominance hémisphérique gauche du langage disparaît.

Mais dès neuf mois après l'opération, l'articulation est redevenue une nouvelle habitude relatéralisée du côté du langage, comme le montre l'activité de l'insula gauche, le cervelet n'étant pas encore automatiquement recruté. 
Il est ainsi possible de retrouver les signes d'une reprise de contrôle de «l'incorporation» d'un «nouveau muscle lingual», lus dans l'activité de l'insula, et ceci avant même que la production articulatori-acoustique du sujet ait retrouvé ses qualités préopératoires (notamment, comme le montre par ailleurs une partie de l'étude acoustique, celle sur les consonnes, qui ne sera pas publiée ici). On peut espérer que lorsque l'activité du cervelet aura retrouvé elle aussi son niveau préopératoire, ce sera le signe de l'acquisition maîtrisée d'une nouvelle habileté de la langue avec de nouveaux moyens musculaires. Ce que nous montrera peut-être un jour ce patient, s'il nous est donné de le suivre plus avant.

REMERCIEMENTS : Ils vont tout particulièrement à M. K.H. qui a accepté de se prêter de manière très coopérative à l'ensemble des examens qui ont été nécessaires pour ce travail. Notre souvenir va aussi aux autres patients qui nous ont quittés trop tôt pour que nous puissions les suivre ou qui ont fait beaucoup d'efforts malgré leur condition difficile, et pour lesquels nos efforts communs n'ont pu pour diverses raisons finalement aboutir.

Le présent projet a fait l'objet d'un appel à propositions du CNRS : ACI (Action Concertée Incitative) Cognitique sur le thème : Perturbations et récupération des fonctions cognitives $d u$ sujet normal et du sujet pathologique. Il a été retenu avec le numéro COG 75 sous le titre: Production de la parole en présence de perturbations fonctionnelles, pathologiques ou artificielles : étude des mécanismes de ré-apprentissage et de récupération.

\section{Références bibliographiques}

1. Ackermann H. \& Riecker A. (2004). The contribution of the insula to motor aspects of speech production : A review and a hypothesis. Brain and Language, 89(2), 320-328.

2. Baciu M., Abry C., \& Segebarth C. (2000). Equivalence motrice et dominance hémisphérique, le cas de la voyelle [u]. Etude IRMf. Actes des $23^{\text {es }}$ Journée d'Etude sur la Parole, 213-216.

3. Bennett S., \& Netsell R.W. (1999). Possible roles of the insula in speech and language processing : direction for research. Journal of Medical Speech-Language Pathology, 7(4), 253-270.

4. Calliope (1989). La parole et son traitement automatique. Editions Masson, Paris.

5. Delattre P. (1948). Un triangle acoustique des voyelles orales du français. French Review, 21, 477-484.

6. Devauchelle B., Badet L., Lengelé B., Morelon E., Testelin S., Michallet M., D'Hauthuille C., \& Dubernard J-M. (2006). First human face allograft : Early report, Lancet, 368, 203-209.

7. Dronkers N.F. (1996). A new brain region for coordinating speech articulation. Nature, 384, 159-161.

8. Friston, K. J., Holmes, A. P., Worsley, K. J., Poline, J.-B., Frith, C. D., \& Frackowiak, R.S.J. (1995). Statistical parametric maps in functional imaging: A general linear approach. Human Brain Mapping, 2, 189-210.

9. Gay, T., Lindblom, B., \& Lubker, J. (1981). Production of bite-block vowels : Acoustic equivalence by selective compensation. Journal of the Acoustical Society of America, 69, pages $802-810$. 
10. Gay T., Boë L.J., \& Perrier P. (1993). Acoustic and perceptual effects of changes in vocal tract constrictions for vowels. Journal of the Acoustical Society of America, 92(3), 1301-1309.

11. Giraux P., Sirigu A., Schneider F., \& Dubernard J-M. (2001). Cortical reorganization in motor cortex after graft of both hands. Nature Neuroscience, 4 (7), 691-692.

12. Gloor P. (1997). The Temporal Lobe and the Limbic System. Oxford University Press, Oxford.

13. Guenther F.H., Ghosh S.S., Nieto-Castanon A., \& Tourville J.A. (2006). A neural model of speech production. In J. Harrington \& M. Tabain (Eds), Speech Production. Models, Phonetic Processes, and Techniques. Psychology Press, New York, 27-39.

14. Hillis A.E., Work M., Barker P. B., Jacobs M. A., Breese E. L., \& Maurer K. (2004). Re-examining the brain regions crucial for orchestrating speech articulation. Brain, 127, $1479-1487$.

15. Houde, J., \& Jordan, M. (1998). Sensorimotor adaptation in speech production. Science, 279 (5354), 1213-1216.

16. Ivry R.B., Justus T.C., \& Middleton C. (2001). The cerebellum, timing, and language : implications for the study of dyslexia. In M. Wolf (Ed.), Dyslexia, Fluency, and the Brain. York Press, Timonium (MD), 189-211.

17. Jones J.A., \& Munhall K.G. (2002). Learning to produce speech with an altered vocal tract : the role of auditory feedback. Journal of the Acoustical Society of America, 113 (1), 532-543.

18. Ogar J., Willock S., Baldo J., Wilkins D., Ludy C., \& Dronkers N. (2006). Clinical and anatomical correlates of apraxia of speech. Brain and Language, 97(3), 343-350 [Epub 2006 Mar 6].

19. Riecker A., Ackermann H., Wildgruber D., Dogil G., \& Grodd W. (2000). Opposite hemispheric lateralization effects during speaking and singing at motor cortex, insula and cerebellum. Neuroreport, 11, 1997-2000.

20. Savariaux C., Perrier P., \& Orliaguet J.P. (1995). Compensation strategies for the perturbation of the rounded vowel $[\mathrm{u}]$ using a lip tube : A study of the control space in speech production. Journal of the Acoustical Society of America, 98 (5), 2428-2442.

21. Savariaux C., Perrier P., Orliaguet J.P., \& Schwartz J.L. (1999). Compensation strategies for the perturbation of French [u] using a lip tube. II. Perceptual analysis. Journal of the Acoustical Society of America, 106 (1), 381-393.

22. Schwartz J.-L., Beautemps D., Abry C., \& Escudier P. (1992). Inter-individual and cross-linguistic strategies for the production of the [i] vs [y] contrast. Journal of Phonetics, 21, 441-445.

23. Wise R.J., Green J., Buchel C., \& Scott S.K (1999). Brain regions involved in articulation. Lancet 353, 1057-1061. 